\title{
WEIGHTED NORM INEQUALITIES FOR GENERAL OPERATORS ON MONOTONE FUNCTIONS
}

\author{
SHANZHONG LAI
}

ABstract. In this paper we characterize the weights $w, v$ for which $\left\|S_{\phi} f\right\|_{p, w}$ $\leq C\|f\|_{q, v}$, for $f$ nonincreasing, where $S_{\phi} f=\int_{0}^{\infty} \phi(x, y) f(y) d y$.

\section{INTRODUCTION}

In this paper we will study weighted norm inequalities of general operators of the form

$$
S_{\phi} f(x)=\int_{0}^{\infty} \phi(x, y) f(y) d y
$$

on monotone functions $f: \mathbb{R}_{+} \rightarrow \mathbb{R}_{+}$. Operators of this type dominate many classical operators $T$ in the sense that $(T f)^{*}(t) \leq C S_{\phi} f^{*}(t)$, where $g^{*}(t)=$ $\inf \{y ;|\{x ;|g(x)|>y\}| \leq t\}$, the rearrangement of $g$. We refer the reader to $[2,5]$ for examples, as the Hardy-Littlewood maximal operator, the Hilbert transform, etc.

It is thus of interest to characterize the weights $w: \mathbb{R}_{+} \rightarrow \mathbb{R}_{+}$for which

$$
\left\|S_{\phi} f\right\|_{p, w} \leq C\|f\|_{p, w},
$$

as this gives extensions of the classical norm inequalities. This is the reason why the study of (1.2) has recently attracted a great deal of attention [3, 4, 6-9], beginning with [1] Ariño and Muckenhoupt for the averaging operator $A f(x)=\frac{1}{x} \int_{0}^{x} f$ to the more general version of [3] for operators of the type $S_{\phi} f(x)=\int_{0}^{1} \phi(t) f(t x) d t$. All of these operators are special cases of (1.1). In this paper we use extensions and refinements of the method introduced in [6] for $A f$ to characterize those $w: \mathbb{R}_{+} \rightarrow \mathbb{R}_{+}$for which (1.2) holds for monotone functions. This will be done $\S 2-\S 6$. The final section deals with applications and a discussion of the sharp norm constant in (1.2) for various choices of $\phi: \mathbb{R}_{+} \times \mathbb{R}_{+} \rightarrow \mathbb{R}_{+}$and $w: \mathbb{R}_{+} \rightarrow \mathbb{R}_{+}$.

Throughout we shall use the notation $f \downarrow(f \uparrow)$ to indicate that $f: \mathbb{R}_{+} \rightarrow$ $\mathbb{R}_{+}$is nonincreasing (nondecreasing). When proving inequalities for monotone functions, we may as usual restrict ourselves to homeomorphisms since a general monotone function can be approximated by homeomorphisms.

Received by the editors September 25, 1990 and, in revised form, October 15, 1991. 1991 Mathematics Subject Classification. Primary 26D15, 42B25.

Key words and phrases. Weighted inequalities, strong type inequality, general operator. Research supported in part by a grant from Purdue University Research Foundation. 
Acknowledgment. The author would like to thank Professor C. J. Neugebauer for many valuable comments and helpful discussions.

\section{Operator $S_{\phi}$}

In this section we study $p, q$-norm inequalities with double weights for

$$
S_{\phi} f(x)=\int_{0}^{\infty} \phi(x, y) f(y) d y .
$$

Define

$$
\Phi(x, r)=\int_{0}^{r} \phi(x, y) d y, \quad \Phi_{1}(x, r)=\int_{r}^{\infty} \phi(x, y) d y,
$$

where $\phi: \mathbb{R}_{+} \times \mathbb{R}_{+} \longrightarrow \mathbb{R}_{+}$. We have

Theorem 2.1. If $1 \leq q \leq p<\infty$, then

$$
\left(\int_{0}^{\infty} f^{p} w\right)^{1 / p} \leq C\left[\int_{0}^{\infty}\left(S_{\phi} f\right)^{q} v\right]^{1 / q}
$$

holds for all $f \downarrow$ iff

$$
\left(\int_{0}^{r} w\right)^{1 / p} \leq C\left[\int_{0}^{\infty} \Phi(x, r)^{q} v\right]^{1 / q}, \quad \forall r>0 .
$$

Moreover (2.1) and (2.2) have same constant $C$.

Theorem 2.2. If $0<q \leq p \leq 1$, then

$$
\left[\int_{0}^{\infty}\left(S_{\phi} f\right)^{p} w\right]^{1 / p} \leq C\left(\int_{0}^{\infty} f^{q} v\right)^{1 / q}
$$

holds for all $f \downarrow$ iff

$$
\left[\int_{0}^{\infty} \Phi(x, r)^{p} w\right]^{1 / p} \leq C\left(\int_{0}^{r} v\right)^{1 / q}, \quad \forall r>0 .
$$

Moreover (2.3) and (2.4) have same constant $C$.

Theorem 2.3. If $1 \leq q \leq p<\infty$, then (2.1) holds for all $f \uparrow$ iff

$$
\left(\int_{r}^{\infty} w\right)^{1 / p} \leq C\left[\int_{0}^{\infty} \Phi_{1}(x, r)^{q} v\right]^{1 / q}, \quad \forall r>0 .
$$

Moreover (2.1) and (2.5) have same constant $C$.

Theorem 2.4. If $0<q \leq p \leq 1$, then (2.3) holds for all $f \uparrow$ iff

$$
\left(\int_{0}^{\infty} \Phi_{1}(x, r)^{p} w\right)^{1 / p} \leq C\left(\int_{r}^{\infty} v\right)^{1 / q}, \quad \forall r>0 .
$$

Moreover (2.3) and (2.6) have same constant $C$.

To prove Theorems 2.1-2.4, we need the following lemmas. 
Lemma 2.5. Suppose $g, h: \mathbb{R}_{+} \rightarrow \mathbb{R}_{+}, h$ is $A C$, and $h^{\prime} \geq 0, h\left(0^{+}\right)=0$, then

(i) For $q \geq 1$,

$$
\int_{0}^{r} g \leq C_{0} h(r)^{q}, \quad \forall r>0
$$

iff

$$
\int_{0}^{\infty} f^{q} g \leq C_{0}\left[\int_{0}^{\infty} f h^{\prime}\right]^{q}, \quad \forall f \downarrow .
$$

(ii) For $0<q \leq 1$,

$$
\int_{0}^{r} g \geq C_{0} h(r)^{q}, \quad \forall r>0
$$

iff

$$
\int_{0}^{\infty} f^{q} g \geq C_{0}\left[\int_{0}^{\infty} f h^{\prime}\right]^{q}, \quad \forall f \downarrow .
$$

Lemma 2.6. Suppose $h$ is $A C$, and $h^{\prime} \leq 0, h\left(\infty^{-}\right)=0$, then

(i) For $q \geq 1$,

$$
\int_{r}^{\infty} g \leq C_{0} h(r)^{q}, \quad \forall r>0
$$

iff

(ii) For $0<q \leq 1$,

$$
\int_{0}^{\infty} f^{q} g \leq C_{0}\left[-\int_{0}^{\infty} f h^{\prime}\right]^{q}, \quad \forall f \uparrow .
$$

$$
\int_{r}^{\infty} g \geq C_{0} h(r)^{q}, \quad \forall r>0
$$

iff

$$
\int_{0}^{\infty} f^{q} g \geq C_{0}\left[-\int_{0}^{\infty} f h^{\prime}\right]^{q}, \quad \forall f \uparrow .
$$

Proof of Lemma 2.5. Suppose $q \geq 1$.

$(2.8) \rightarrow(2.7)$ Let $f=\chi_{(0, r)}$.

$(2.7) \rightarrow(2.8)$ Let $r=\psi(y) \downarrow, \psi(0)=\infty, \psi(\infty)=0$, then

$$
\begin{array}{r}
\int_{0}^{\infty}\left[\int_{0}^{\psi(y)} g(t) d t\right]^{1 / q} d y \leq C_{0}^{1 / q} \int_{0}^{\infty} h(\psi(y)) d y \\
=-C_{0}^{1 / q} \int_{0}^{\infty} h(t) d \psi^{-1}(t) \\
=C_{0}{ }^{1 / q} \int_{0}^{\infty} \psi^{-1}(t) h^{\prime}(t) d t
\end{array}
$$

LHS $=\int_{0}^{\infty}\left[\int_{0}^{\infty} \chi_{(0, \psi(y))}^{q}(t) g(t) d t\right]^{1 / q} d y$

$$
\begin{aligned}
& \geq\left\{\int_{0}^{\infty}\left[\int_{0}^{\infty} \chi_{(0, \psi(y))}(t) d y\right]^{q} g(t) d t\right\}^{1 / q} \text { by Minkowski's inequality } \\
& =\left[\int_{0}^{\infty} \psi^{-1}(t)^{q} g(t) d t\right]^{1 / q}
\end{aligned}
$$


Now let $\psi^{-1}(t)=f(t)$ to complete the proof for (i). The proof for (ii) is similar.

The proof of Lemma 2.6 is similar to that of Lemma 2.5. In fact we can show that Lemma 2.5 and Lemma 2.6 are equivalent. For convenience we state the following particular case of Lemma 2.5 , which can be derived by taking $g(x)=x^{p-1}, h(r)=r, C_{0}=1 / p$.

Lemma 2.7. (i) For $0<p \leq 1$, we have

$$
\left(\int_{0}^{\infty} f\right)^{p} \leq p \int_{0}^{\infty} f^{p}(x) x^{p-1} d x, \quad \forall f \downarrow .
$$

(ii) For $p \geq 1$, we have

$$
\left(\int_{0}^{\infty} f\right)^{p} \geq p \int_{0}^{\infty} f^{p}(x) x^{p-1} d x, \quad \forall f \downarrow
$$

Proof of Theorem 2.1. (2.1) $\rightarrow(2.2)$ Let $f=\chi_{(0, r)}$.

$(2.2) \rightarrow(2.1)$ Let $r=\psi(y) \downarrow$, where $\psi:(0, \infty) \rightarrow(0, \infty)$ is onto, then

$$
\begin{aligned}
L & \equiv \int_{0}^{\infty}\left(\int_{0}^{\psi(y)} w(x) d x\right)^{q / p} y^{q / p-1} d y \\
& \leq C^{q} \int_{0}^{\infty} \int_{0}^{\infty} \Phi(x, \psi(y))^{q} v(x) d x y^{q / p-1} d y \\
& \equiv C^{q} R
\end{aligned}
$$

where

$$
\begin{aligned}
R & =\int_{0}^{\infty} \int_{0}^{\infty} \Phi(x, \psi(y))^{q} y^{q / p-1} d y v(x) d x \\
& \equiv \int_{0}^{\infty} I(x) v(x) d x
\end{aligned}
$$

Fix $x>0$, let $t=\psi(y)$ in $I(x)$, then

$$
\begin{aligned}
I(x) & =-\int_{0}^{\infty} \Phi(x, t)^{q} \psi^{-1}(t)^{q / p-1} d \psi^{-1}(t) \\
& =\frac{p}{q} \cdot q \int_{0}^{\infty} \psi^{-1}(t)^{q / p} \Phi(x, t)^{q-1} \phi(x, t) d t .
\end{aligned}
$$

Now take

$$
\begin{aligned}
& g(t)=\Phi(x, t)^{q-1} \phi(x, t), \quad h(t)=\Phi(x, t), \\
& f(t)=\psi^{-1}(t)^{1 / p}, \quad C_{0}=\frac{1}{q}
\end{aligned}
$$

in Lemma 2.5(i). We get

$$
I(x) \leq \frac{p}{q}\left[\int_{0}^{\infty} \psi^{-1}(t)^{1 / p} \phi(x, t) d t\right]^{q} .
$$


Now by Lemma $2.7(\mathrm{i})$, since $q \leq p$,

$$
\begin{aligned}
L & \geq \frac{p}{q}\left[\int_{0}^{\infty} \int_{0}^{\psi(y)} w(x) d x d y\right]^{q / p} \\
& =\frac{p}{q}\left[\int_{0}^{\infty} \psi^{-1}(x) w(x) d x\right]^{q / p} .
\end{aligned}
$$

Finally by taking $\psi^{-1}(x)=f(x)^{p}$, we complete the proof with the same constant $C$.

Proof of Theorem 2.2. (2.3) $\rightarrow(2.4)$ Let $f=\chi_{(0, r)}$.

$(2.4) \rightarrow(2.3)$ Let $r=\psi(y) \downarrow$, then

$$
\begin{aligned}
L & =\int_{0}^{\infty} \int_{0}^{\infty} \Phi(x, \psi(y))^{p} w(x) d x y^{p / q-1} d y \\
& \leq C^{p} \int_{0}^{\infty}\left(\int_{0}^{\psi(y)} v(x) d x\right)^{p / q} y^{p / q-1} d y \\
& \leq C^{p} \frac{q}{p}\left[\int_{0}^{\infty} \int_{0}^{\psi(y)} v(x) d x d y\right]^{p / q} \quad \text { by Lemma } 2.7(\mathrm{ii}) \\
& =C^{p} \frac{q}{p}\left[\int_{0}^{\infty} \psi^{-1}(x) v(x) d x\right]^{p / q}, \\
L & =\int_{0}^{\infty} \int_{0}^{\infty} \Phi(x, \psi(y))^{p} y^{p / q-1} d y w(x) d x .
\end{aligned}
$$

Denote

$$
I(x)=\int_{0}^{\infty} \Phi(x, \psi(y))^{p} y^{p / q-1} d y
$$

Let $t=\psi(y)$, then

$$
\begin{aligned}
I(x) & =-\int_{0}^{\infty} \Phi(x, t)^{p} \psi^{-1}(t)^{p / q-1} d \psi^{-1}(t) \\
& =\frac{q}{p} p \int_{0}^{\infty} \psi^{-1}(t)^{p / q} \Phi(x, t)^{p-1} \phi(x, t) d t \\
& \geq \frac{q}{p}\left[\int_{0}^{\infty} \psi^{-1}(t)^{1 / q} \phi(x, t) d t\right]^{p} \quad \text { by Lemma } 2.5(\mathrm{ii}) .
\end{aligned}
$$

Finally take $\psi^{-1}(t)=f(t)^{q}$.

The proofs of Theorems 2.3 and 2.4 are similar to those of Theorems 2.1 and 2.2. In fact we can show that Theorem 2.1 and Theorem 2.3, Theorem 2.2 and Theorem 2.4 are equivalent respectively by the following change of variable,

$$
S_{\phi} f(x)=\int_{0}^{\infty} \frac{\phi(x, 1 / t)}{t^{2}} \widetilde{f}(t) d t
$$


with $\tilde{f}(t)=f(1 / t) \downarrow$ if $f \uparrow$ and so on. In $\S 7$ we will give some applications of these theorems.

\section{Operator $T_{\phi}$}

In order to obtain $\left\|S_{\phi} f\right\|_{p, w} \leq C\|f\|_{p, w}$ in the range $1 \leq p<\infty$, it is convenient to split $S_{\phi}=T_{\phi}+T_{\phi}^{*}$, where

$$
T_{\phi} f(x)=\int_{0}^{x} \phi(x, y) f(y) d y \text {. }
$$

The operator $T_{\phi}^{*}$ will be studied in the next section. We shall assume

$$
\begin{gathered}
\Phi(x, r) \leq B \Phi(x, t) \Phi(t, r), \quad 0<r \leq t \leq x ; \\
f \downarrow \Rightarrow T_{\phi} f \downarrow .
\end{gathered}
$$

Remark 1. (H1) implies $\Phi(x, x) \leq B \Phi(x, x)^{2}$ or $\Phi(x, x) \geq B^{-1}$. Also we notice that (H2) is equivalent to the condition $\Phi(x, r) \downarrow$ in $x$ for $x>$ $r$ and $\Phi(x, x) \downarrow$. In fact if the latter condition holds, then for $f \downarrow$, we have $T_{\phi} f(x)=\int_{0}^{x} \phi(x, y) \int_{0}^{\infty} \chi_{E}(y, t) d t d y=\int_{0}^{\infty} \int_{0}^{x} \phi(x, y) \chi_{E}(y, t) d y d t=$ $\int_{0}^{\infty} T_{\phi} \chi_{(0, \tilde{y}(t))}(x) d t \downarrow$, where $E \equiv\{(y, t) ; f(y)>t\}$, and $(0, \tilde{y}(t)) \equiv\{y ; f(y)$ $>t\}$, for $t>0$.

Remark 2. A special case of (H1) already appears in [3].

Theorem 3.1. If $1 \leq p<\infty$, and $(\mathrm{H} 1)$, ( $\mathrm{H} 2)$ hold, then

$$
\int_{0}^{\infty}\left(T_{\phi} f\right)^{p} w \leq C_{1} \int_{0}^{\infty} f^{p} w, \quad \forall f \downarrow
$$

iff

$$
\int_{0}^{r} \Phi(x, x)^{p} w(x) d x+\int_{r}^{\infty} \Phi(x, r)^{p} w(x) d x \leq C_{2} \int_{0}^{r} w, \quad \forall r>0 .
$$

Remark 3. We will see later (Remark after Theorem 6.1) that (H2) plus (3.2) without (H1) is not enough for the norm inequality (3.1) to hold.

Proof. (3.1) $\rightarrow(3.2)$ Let $f=\chi_{(0, r)}$. Then

$$
T_{\phi} f(x)= \begin{cases}\Phi(x, x) & \text { if } x<r \\ \Phi(x, r) & \text { if } x \geq r .\end{cases}
$$

So (3.2) holds with $C_{2}=C_{1}$.

$(3.2) \rightarrow(3.1)$ Let $r=\psi(y) \downarrow$, then

$$
\begin{aligned}
L & \equiv \int_{0}^{\infty} \int_{0}^{\psi(y)} \Phi(x, x)^{p} w(x) d x d y+\int_{0}^{\infty} \int_{\psi(y)}^{\infty} \Phi(x, \psi(y))^{p} w(x) d x d y \\
& \leq C_{2} \int_{0}^{\infty} \int_{0}^{\psi(y)} w(x) d x d y=C_{2} \int_{0}^{\infty} \psi^{-1}(x) w(x) d x .
\end{aligned}
$$

Changing the order of integration and integrating by parts, we get

$$
\begin{aligned}
L & =\int_{0}^{\infty} \psi^{-1}(x) \Phi(x, x)^{p} w(x) d x+\int_{0}^{\infty} \int_{\psi^{-1}(x)}^{\infty} \Phi(x, \psi(y))^{p} d y w(x) d x \\
& =-p \int_{0}^{\infty} \int_{\psi^{-1}(x)}^{\infty} y \Phi(x, \psi(y))^{p-1} \phi(x, \psi(y)) d \psi(y) w(x) d x .
\end{aligned}
$$


Let $t=\psi(y)$, then

$$
L=p \int_{0}^{\infty} \int_{0}^{x} \psi^{-1}(t) \Phi(x, t)^{p-1} \phi(x, t) d t w(x) d x .
$$

Take

$$
\psi^{-1}(t)=f(t)\left(T_{\phi} f\right)^{p-1}(t)=f(t)\left(\int_{0}^{t} \phi(t, y) f(y) d y\right)^{p-1}
$$

then since $(\mathrm{H} 1)$ implies

$$
\int_{0}^{t} \phi(x, y) f(y) d y \leq B \Phi(x, t) \int_{0}^{t} \phi(t, y) f(y) d y,
$$

we get

$$
\begin{aligned}
L & \geq p B^{-(p-1)} \int_{0}^{\infty} \int_{0}^{x}\left(\int_{0}^{t} \phi(x, y) f(y) d y\right)^{p-1} f(t) \phi(x, t) d t w(x) d x \\
& =B^{-(p-1)} \int_{0}^{\infty}\left(\int_{0}^{x} \phi(x, y) f(y) d y\right)^{p} w(x) d x
\end{aligned}
$$

Combining (3.4), (3.5) we complete the proof with $C_{1}=C_{2}^{p} B^{p(p-1)}$ by Hölder's inequality.

Definition. For $0<p<\infty$,

$$
w \in B_{p}(\phi) \leftrightarrow \int_{r}^{\infty} \Phi(x, r)^{p} w(x) d x \leq C \int_{0}^{r} w, \quad \forall r>0 .
$$

Remark 4. If we assume

$$
\Phi(x, x) \leq C,
$$

then we have $B_{q}(\phi) \subset B_{p}(\phi), q \leq p$. The next theorem gives a result for $q>p$.

Remark 5. It is easy to see that if $\eta \downarrow$, then $w \in B_{p}(\phi)$ implies $\eta w \in B_{p}(\phi)$.

Theorem 3.2. Suppose $0<p<\infty$, and (H1), (H2), and (H3) hold, then

$$
w \in B_{p}(\phi) \rightarrow \exists \epsilon>0, \text { such that } w \in B_{p-\epsilon}(\phi) .
$$

Proof. Suppose $w \in B_{p}(\phi)$, then we have by Theorems 2.2 and 3.1,

$$
\int_{0}^{\infty}\left(T_{\phi} f\right)^{p} w \leq C \int_{0}^{\infty} f^{p} w, \quad \forall f \downarrow
$$

Let for $0<\epsilon<1$,

$$
f_{r}(y)= \begin{cases}A, & \text { if } y \leq r, \\ \epsilon \Phi(y, r)^{1-\epsilon}, & \text { if } y>r,\end{cases}
$$

where the constant A will be chosen so that $f_{r} \downarrow$. Then for $y>r, f_{r}(y)=$ 
$\epsilon T_{\phi} \chi_{(0, r)}(y)^{1-\epsilon} \downarrow$ by (H2). Hence for $x>r$,

$$
\begin{aligned}
T_{\phi} f_{r}(x) & =A \int_{0}^{r} \phi(x, y) d y+\epsilon \int_{r}^{x} \phi(x, y) \Phi(y, r)^{1-\epsilon} d y \\
& =A \Phi(x, r)+\epsilon \int_{r}^{x} \phi(x, y) \Phi(y, r)^{1-\epsilon} d y \\
& \geq A \Phi(x, r)+\epsilon B^{1-\epsilon} \int_{r}^{x} \phi(x, y) \frac{\Phi(x, r)^{1-\epsilon}}{\Phi(x, y)^{1-\epsilon}} d y \\
& \geq\left(A-C_{0}\right) \Phi(x, r)+C_{0} \Phi(x, x)^{\epsilon} \Phi(x, r)^{1-\epsilon} \\
& \geq C_{0} \Phi(x, x)^{\epsilon} \Phi(x, r)^{1-\epsilon} \text { if } A>C_{0} \\
& \geq C \Phi(x, r)^{1-\epsilon} \quad \text { since } \Phi(x, x) \geq B^{-1}
\end{aligned}
$$

where $C_{0}=\min \{1, B\}$. We get

$$
\int_{0}^{\infty}\left(T_{\phi} f_{r}\right)^{p} w \geq C \int_{r}^{\infty} \Phi(x, r)^{(1-\epsilon) p} w(x) d x .
$$

On the other hand,

$$
\int_{0}^{\infty} f_{r}^{p} w=A^{p} \int_{0}^{r} w(x) d x+\epsilon^{p} \int_{r}^{\infty} \Phi(x, r)^{(1-\epsilon) p} w(x) d x .
$$

For $\epsilon>0$ small we get $w \in B_{(1-\epsilon) p}(\phi)$.

\section{ADJOINT OPERATOR $T_{\phi}^{*}$}

In this section we consider

$$
T_{\phi}^{*} f(x)=\int_{x}^{\infty} \phi(x, y) f(y) d y,
$$

and set

$$
\Phi^{*}(x, r)=\int_{x}^{r} \phi(x, y) d y+1, \quad x \leq r .
$$

We need conditions similar to (H1), (H2), i.e.

$$
\begin{aligned}
\Phi^{*}(x, y) \leq B \Phi^{*}(x, t) \Phi^{*}(t, y), & \text { for } x \leq t \leq y ; \\
f \downarrow & \Rightarrow T_{\phi}^{*} f \downarrow .
\end{aligned}
$$

We notice that (H5) is equivalent to the condition $\Phi^{*}(x, r) \downarrow$ in $x$ for $x<r, \forall r>0$.

Theorem 4.1. Suppose $\phi$ satisfies (H4), (H5), then for $p \geq 1$,

$$
\int_{0}^{\infty}\left(T_{\phi}^{*} f\right)^{p} w \leq C \int_{0}^{\infty} f^{p} w, \quad \forall f \downarrow
$$

iff

$$
\int_{0}^{r} \Phi^{*}(x, r)^{p} w \leq C \int_{0}^{r} w, \quad \forall r>0 .
$$

Remark. We will see later (Remark after Theorem 6.1) that (H5) plus (4.2) without (H4) is not enough for (4.1) to hold. 
Proof. (4.1) $\rightarrow$ (4.2) Let $f=\chi_{(0, r)}$.

$(4.2) \rightarrow(4.1)$ Let $r=\psi(y) \downarrow$, then

$$
\begin{aligned}
L & \equiv \int_{0}^{\infty} \int_{0}^{\psi(y)} \Phi^{*}(x, \psi(y))^{p} w(x) d x d y \\
& \leq C \int_{0}^{\infty} \int_{0}^{\psi(y)} w(x) d x d y \\
& =C \int_{0}^{\infty} \psi^{-1}(x) w(x) d x \\
& \equiv C R,
\end{aligned}
$$

Changing the order of integration in the definition of $L$, we get

$$
L=\int_{0}^{\infty} \int_{0}^{\psi^{-1}(x)} \Phi^{*}(x, \psi(y))^{p} d y w(x) d x
$$

Let

$$
I(x)=\int_{0}^{\psi^{-1}(x)} \Phi^{*}(x, \psi(y))^{p} d y .
$$

Fix $x>0$, let $u=\Phi^{*}(x, \psi(y)), y=\psi^{-1}\left(\Phi_{x}^{*-1}(u)\right)$. Then

$$
I \equiv I(x)=-\int_{\Phi^{*}(x, x)}^{\infty} u^{p} d \psi^{-1}\left(\Phi_{x}^{*-1}(u)\right)
$$

Let $t=\Phi_{x}^{*-1}(u)$, or $u=\Phi^{*}(x, t)$, we have

$$
I=\left.y \Phi^{*}(x, \psi(y))^{p}\right|_{0} ^{\psi^{-1}(x)}+p \int_{x}^{\infty} \psi^{-1}(t) \Phi^{*}(x, t)^{p-1} \phi(x, t) d t .
$$

Now take

$$
\begin{aligned}
\psi^{-1}(t) & =f(t)\left[T_{\phi}^{*} f(t)+f(t)\right]^{p-1} \\
& =f(t)\left[\int_{t}^{\infty} \phi(t, y) f(y) d y+f(t)\right]^{p-1} .
\end{aligned}
$$

Since (H4) implies for $x \leq t, y>0$,

$$
\int_{t}^{\infty} \phi(x, s) \chi_{(0, y)}(s) d s \leq B \Phi^{*}(x, t)\left(\int_{t}^{\infty} \phi(t, s) \chi_{(0, y)}(s) d s+\chi_{(0, y)}(t)\right),
$$

we have by a suitable approximation argument that

$$
\int_{t}^{\infty} \phi(x, y) f(y) d y \leq B \Phi^{*}(x, t)\left(\int_{t}^{\infty} \phi(t, y) f(y) d y+f(t)\right) .
$$

Thus we get

$$
\begin{aligned}
I & \geq p \int_{x}^{\infty}\left[\int_{t}^{\infty} \phi(t, y) f(y) d y+f(t)\right]^{p-1} \Phi^{*}(x, t)^{p-1} \phi(x, t) f(t) d t \\
& \geq B^{-p+1} p \int_{x}^{\infty}\left[\int_{t}^{\infty} \phi(x, y) f(y) d y\right]^{p-1} \phi(x, t) f(t) d t \\
& =B^{-p+1}\left(T_{\phi}^{*} f\right)^{p}
\end{aligned}
$$


On the other hand

$$
R \leq C \int_{0}^{\infty}\left(\frac{1}{\epsilon} f\right)\left(\epsilon\left(T_{\phi}^{*} f\right)^{p-1}\right) w+C \int_{0}^{\infty} f^{p} w .
$$

Combining this with (4.3)-(4.6), we complete the proof by using Young's inequality with $\epsilon$ small.

Definition. For $0<p<\infty$,

$$
w \in B_{p}^{*}(\phi) \leftrightarrow \int_{0}^{r} \Phi^{*}(x, r)^{p} w(x) d x \leq C \int_{0}^{r} w, \quad \forall r>0 .
$$

If $\phi(x, y) \equiv \frac{1}{x} \chi_{(x, \infty)}(y)$, we simply write $B_{p}^{*}$ instead of $B_{p}^{*}(\phi)$.

Corollary 4.2. Suppose $0<p<\infty$, and if $p>1, \phi$ satisfies (H1)-(H5), then

$$
\int_{0}^{\infty}\left(T_{\phi} f+T_{\phi}^{*} f\right)^{p} w \leq C \int_{0}^{\infty} f^{p} w
$$

iff

$$
w \in B_{p}(\phi) \cap B_{p}^{*}(\phi) .
$$

Remark. Suppose (H5) holds and $\eta \uparrow$, then $w \in B_{p}^{*}(\phi)$ implies $\eta w \in B_{p}^{*}(\phi)$. Proof. Let $0<s<r, w \in B_{p}^{*}(\phi)$, then

$$
\int_{0}^{r} \Phi^{*}(x, r)^{p} w(x) d x \leq C_{0} \int_{0}^{r} w(x) d x .
$$

So, if $\Phi^{*}(s, r)^{p} \geq C_{0}$, we have by (H5)

$$
\begin{aligned}
\int_{s}^{r} \Phi^{*}(x, r)^{p} w & =\int_{0}^{r} \Phi^{*}(x, r)^{p} w-\int_{0}^{s} \Phi^{*}(x, r)^{p} w \\
& \leq C_{0} \int_{0}^{r} w-\Phi^{*}(s, r)^{p} \int_{0}^{s} w \\
& \leq C_{0} \int_{s}^{r} w .
\end{aligned}
$$

If $\Phi^{*}(s, r)^{p} \leq C_{0}$, then

$$
\int_{s}^{r} \Phi^{*}(x, r)^{p} w \leq \Phi^{*}(s, r)^{p} \int_{s}^{r} w \leq C_{0} \int_{s}^{r} w
$$

Thus

$$
\int_{s}^{r} \Phi^{*}(x, r)^{p} w \leq C_{0} \int_{s}^{r} w, \quad \forall 0<s<r .
$$

From this we get

$$
\int_{0}^{r} \Phi^{*}(x, r)^{p} \eta w \leq C_{0} \int_{0}^{r} \eta w, \quad \forall \eta \uparrow
$$

by a suitable approximation argument.

It is clear that $B_{q}^{*}(\phi) \subset B_{p}^{*}(\phi), q \geq p$, and in the other direction we have 
Theorem 4.3. Suppose $0<p<\infty$, and (H4), (H5) hold. Then

$$
w \in B_{p}^{*}(\phi) \rightarrow \exists \epsilon>0 \text {, such that } w \in B_{p+\epsilon}^{*}(\phi) .
$$

Proof. By Theorems 2.1 and 4.1, we have

$$
\int_{0}^{\infty}\left(T_{\phi}^{*} f\right)^{p} w \leq C \int_{0}^{\infty} f^{p} w, \quad \forall f \downarrow .
$$

Take

$$
f(x)=(\alpha-1) \Phi^{*}(x, r)^{\alpha} \chi_{(0, r)}(x), \quad \alpha>1 \text { to be chosen. }
$$

Then for $x \leq r$,

$$
\begin{aligned}
T_{\phi}^{*} f(x) & =(\alpha-1) \int_{x}^{r} \Phi^{*}(y, r)^{\alpha} \phi(x, y) d y \\
& \geq \frac{\alpha-1}{B^{\alpha}} \Phi^{*}(x, r)^{\alpha} \int_{x}^{r} \frac{\phi(x, y)}{\Phi^{*}(x, y)^{\alpha}} d y \quad \text { by }(\mathrm{H} 4) \\
& =\left.\frac{1}{B^{\alpha}} \Phi^{*}(x, r)^{\alpha} \Phi^{*}(x, y)^{1-\alpha}\right|_{y=r} ^{x} \\
& =\frac{1}{B^{\alpha}}\left[\Phi^{*}(x, x)^{1-\alpha} \Phi^{*}(x, r)^{\alpha}-\Phi^{*}(x, r)\right] .
\end{aligned}
$$

So

$$
\begin{aligned}
\int_{0}^{r} & \Phi^{*}(x, x)^{(1-\alpha) p} \Phi^{*}(x, r)^{\alpha p} w(x) d x \\
& \leq C \int_{0}^{r} \Phi^{*}(x, r)^{p} w(x) d x+(\alpha-1)^{p} C^{\prime}(p, B) \int_{0}^{r} \Phi^{*}(x, r)^{\alpha p} w(x) d x \\
& \leq C \int_{0}^{r} w+(\alpha-1)^{p} C^{\prime} \int_{0}^{r} \Phi^{*}(x, r)^{\alpha p} w(x) d x .
\end{aligned}
$$

Note that $\Phi^{*}(x, x)=1$ and $w \in B_{p}^{*}(\phi)$. Choosing $\alpha$ close to 1 , we get $w \in B_{\alpha p}(\phi)$.

Corollary 4.4. Under the hypothesis of Theorem 4.3, we have for some $\epsilon>0$,

$$
\Phi^{*}(r, \sigma r)^{p+\epsilon} \frac{\int_{0}^{r} w}{\int_{0}^{\sigma r} w} \leq C, \quad \forall r>0, \sigma>1 .
$$

Proof. $w \in B_{p}^{*}(\phi) \rightarrow \exists \epsilon>0$ such that $w \in B_{p+\epsilon}^{*}(\phi)$, so

$$
\int_{0}^{r} \Phi^{*}(x, r)^{p+\epsilon} w(x) d x \leq C \int_{0}^{r} w(x) d x .
$$

Let $\sigma \geq 1$,

$$
\begin{aligned}
\int_{0}^{r} w(x) d x & =\int_{0}^{r} \frac{\Phi^{*}(x, \sigma r)^{p+\epsilon}}{\Phi^{*}(x, \sigma r)^{p+\epsilon}} w(x) d x \\
& \leq \frac{C}{\Phi^{*}(r, \sigma r)^{p+\epsilon}} \int_{0}^{\sigma r} w
\end{aligned}
$$

since $\Phi^{*}(x, r) \downarrow$ in $x$ by $(\mathrm{H} 5)$. 


\section{CALDERÓN OPERATOR}

In this section we consider Calderón operators

$$
\begin{aligned}
T f(x) & =x^{-\alpha} \int_{0}^{x^{\beta}} s^{\gamma-1} f(s) d s, \quad \alpha, \beta, \gamma>0 ; \\
T^{*} f(x) & =x^{-\alpha_{1}} \int_{x^{\beta}}^{1} s^{\gamma_{1}-1} f(s) d s, \quad \alpha_{1}, \gamma_{1} \geq 0 \\
S f(x) & =T f(x)+T^{*} f(x) .
\end{aligned}
$$

These operators occur in the study of operators which are weak type $\left(p_{i}, q_{i}\right)$, $i=1,2[2]$.

It is easy to see that for $f \downarrow$, if $\beta \leq \alpha / \gamma$ then $T f \downarrow$, and if $-\alpha_{1} \leq \beta \gamma_{1}$, then $T^{*} f \downarrow$. Denote $\delta=\beta \gamma-\alpha$, then we have

Theorem 5.1. Suppose $\delta \leq 0, \alpha \geq \gamma, p \geq 1$, then

$$
\int_{0}^{1}(T f)^{p} w \leq C \int_{0}^{1} f^{p} w, \quad \forall f \downarrow
$$

iff

$$
\int_{0}^{r} x^{p \delta} w+r^{p \delta} \int_{r}^{1}\left(\frac{r}{x}\right)^{p \alpha} w \leq C \int_{0}^{r^{\beta}} w, \quad \forall 0<r<1 .
$$

Proof. (5.1) $\rightarrow$ (5.2) Let $f=\chi_{\left(0, r^{\beta}\right)}$.

$(5.2) \rightarrow(5.1) \quad$ Let $r=\psi(y)^{\beta} \downarrow$, where $\psi:(0, \infty) \rightarrow(0,1)$ is onto. Then

$$
\begin{aligned}
L & \equiv \int_{0}^{\infty} \int_{\psi(y)}^{1}\left(\frac{\psi(y)^{\beta \gamma}}{x^{\alpha}}\right)^{p} w(x) d x d y \\
& \leq C \int_{0}^{\infty} \int_{0}^{\psi(y)^{\beta}} w(x) d x d y \\
& \leq C \int_{0}^{1} \psi^{-1}\left(x^{\frac{1}{\beta}}\right) w(x) d x \\
& \equiv C R .
\end{aligned}
$$

Changing the order of integration, we get

$$
L=\int_{0}^{1} \int_{\psi^{-1}(x)}^{\infty} \psi(y)^{\beta \gamma p} d y \frac{w(x)}{x^{\alpha p}} d x .
$$

Denote

$$
I(x)=\int_{\psi^{-1}(x)}^{\infty} \psi(y)^{\beta \gamma p} d y
$$

and let $u=\psi(y)$, then we have

$$
\begin{aligned}
I & =-\int_{0}^{x} u^{\beta \gamma p} d \psi^{-1}(u) \\
& =-\left.u^{\beta \gamma p} \psi^{-1}(u)\right|_{0} ^{x}+\beta \gamma p \int_{0}^{x} \psi^{-1}(u) u^{\beta \gamma p-1} d u .
\end{aligned}
$$


Let $I_{1}$ be the last integral. Take

$$
\psi^{-1}(u)=f\left(u^{\beta}\right)\left[u^{-\beta \gamma} \int_{0}^{u^{\beta}} s^{\gamma-1} f(s) d s\right]^{p-1}
$$

then

$$
\begin{aligned}
I_{1} & =\int_{0}^{x}\left[\int_{0}^{u^{\beta}} s^{\gamma-1} f(s) d s\right]^{p-1} u^{\beta \gamma-1} f\left(u^{\beta}\right) d u \\
& =\frac{1}{\beta p}\left[\int_{0}^{x^{\beta}} s^{\gamma-1} f(s) d s\right]^{p} .
\end{aligned}
$$

So

$$
\begin{aligned}
L & \geq \gamma \int_{0}^{1}\left[\int_{0}^{x^{\beta}} s^{\gamma-1} f(s) d s\right]^{p} \frac{w(x)}{x^{\alpha p}} d x-\int_{0}^{1} x^{\beta \gamma p} \psi^{-1}(x) w(x) d x \\
& \geq \gamma \int_{0}^{1}\left[\int_{0}^{x^{\beta}} s^{\gamma-1} f(s) d s\right]^{p} \frac{w(x)}{x^{\alpha p}} d x-C R \quad \text { by }(5.2) \\
R & \equiv \int_{0}^{1} f(x)\left[x^{-\gamma} \int_{0}^{x} s^{\gamma-1} f(s) d s\right]^{p-1} w(x) d x .
\end{aligned}
$$

(1) If $\beta \leq 1$, then $x \leq x^{\beta}$. Since $\alpha \geq \gamma$, we have

$$
\begin{aligned}
R & \leq \int_{0}^{1} f(x)\left[x^{-\alpha} \int_{0}^{x^{\beta}} s^{\gamma-1} f(s) d s\right]^{p-1} x^{(\alpha-\gamma)(p-1)} w(x) d x \\
& \leq \int_{0}^{1} f(T f)^{p-1} w .
\end{aligned}
$$

(2) If $\beta \geq 1$, then $x \geq x^{\beta}$. Since $T f \downarrow$ if $f \downarrow$,

$$
x^{-\gamma} \int_{0}^{x} s^{\gamma-1} f(s) d s \leq x^{-\beta \gamma} \int_{0}^{x^{\beta}} s^{\gamma-1} f(s) d s
$$

so that

$$
\begin{aligned}
R & \leq \int_{0}^{1} f(x)(T f)^{p-1}(x) x^{(\alpha-\beta \gamma)(p-1)} w(x) d x \\
& \leq \int_{0}^{1} f(T f)^{p-1} w
\end{aligned}
$$

since $\alpha-\beta \gamma \geq 0$.

Theorem 5.2. Suppose $\beta=\frac{\alpha-\alpha_{1}}{\gamma-\gamma_{1}}, \delta \equiv \beta \gamma-\alpha \quad\left(=\beta \gamma_{1}-\alpha_{1}\right) \leq 0$, and $\beta \geq 1$.

Then for $1 \leq p<\infty$,

$$
\int_{0}^{1}(S f)^{p} w \leq C \int_{0}^{1} f^{p} w, \quad \forall f \downarrow
$$

iff $\forall 0<r<1$,

$$
\int_{0}^{r} x^{p \delta} w+r^{p \delta} \int_{0}^{r}\left(\frac{r}{x}\right)^{p \alpha_{1}} w+r^{p \delta} \int_{r}^{1}\left(\frac{r}{x}\right)^{p \alpha} w \leq C \int_{0}^{r^{\beta}} w .
$$


Proof. (5.3) $\rightarrow$ (5.4) Let $f=\chi_{\left(0, r^{\beta}\right)}$.

$(5.4) \rightarrow(5.3)$ In view of Theorem 5.1 we only need to show the norm inequality holds for $T^{*} f$. Let $r=\psi(y) \downarrow$, where $\psi:(0, \infty) \rightarrow(0,1)$ is onto. Then we have

$$
\begin{aligned}
L & \equiv \int_{0}^{1} I_{1}(x) \frac{w(x)}{x^{\alpha_{1} p}} d x \equiv \int_{0}^{1} \int_{0}^{\psi^{-1}(x)} \psi(y)^{\beta \gamma_{1} p} d y \frac{w(x)}{x^{\alpha_{1} p}} d x \\
& \leq C \int_{0}^{\infty} \int_{0}^{\psi(y)^{\beta}} w(x) d x d y \\
& =C \int_{0}^{1} \psi^{-1}\left(x^{\frac{1}{\beta}}\right) w(x) d x \\
& \equiv C R .
\end{aligned}
$$

(1) Let $u=\psi(y)$ in $I_{1}$, then

$$
\begin{aligned}
I_{1} & =-\int_{x}^{1} u^{\beta \gamma p} d \psi^{-1}(u) \\
& =x^{\beta \gamma_{1} p} \psi^{-1}(x)+\beta \gamma_{1} p \int_{x}^{1} \psi^{-1}(u) u^{\beta \gamma_{1} p-1} d u .
\end{aligned}
$$

Let

$$
\psi^{-1}(u)=f\left(u^{\beta}\right)\left[u^{-\beta \gamma_{1}} \int_{u^{\beta}}^{1} s^{\gamma_{1}-1} f(s) d s\right]^{p-1} .
$$

Since

$$
\frac{d}{d u} \int_{u^{\beta}}^{1} s^{\gamma_{1}-1} f(s) d s=-\beta u^{\beta \gamma_{1}-1} f\left(u^{\beta}\right),
$$

we get

$$
\begin{aligned}
I_{1} & =x^{\beta \gamma_{1} p} \psi^{-1}(x)+\beta \gamma_{1} p \int_{x}^{1}\left[\int_{u^{\beta}}^{1} s^{\gamma_{1}-1} f(s) d s\right]^{p-1} f\left(u^{\beta}\right) u^{\beta \gamma_{1}-1} d u \\
& =x^{\beta \gamma_{1} p} \psi^{-1}(x)+\gamma_{1}\left[\int_{x^{\beta}}^{1} s^{\gamma_{1}-1} f(s) d s\right]^{p} .
\end{aligned}
$$

So

$$
L \geq \gamma_{1} \int_{0}^{1}\left(T^{*} f\right)^{p} w
$$

(2) Now we estimate R.

$$
\begin{aligned}
R & =\int_{0}^{1} f(x)\left[x^{-\gamma_{1}} \int_{x}^{1} s^{\gamma_{1}-1} f(s) d s\right]^{p-1} w(x) d x \\
& \leq \int_{0}^{1} f\left(T^{*} f\right)^{p-1} w
\end{aligned}
$$

since $\gamma_{1} \beta \leq \alpha_{1}, \beta \geq 1$.

If $\gamma_{1}=0, \alpha_{1}>0$, we have 
Theorem 5.3. If $1 \leq p<\infty, 1 \leq \beta=\frac{\alpha-\alpha_{1}}{\gamma}<\frac{\alpha}{\gamma}$, then (5.3) holds iff (5.2) holds.

Proof. In this case

$$
T^{*} f(x)=x^{-\alpha_{1}} \int_{x^{\beta}}^{1} s^{-1} f(s) d s .
$$

So

$$
T^{*} f(x) \leq x^{-\alpha_{1}} f\left(x^{\beta}\right) \beta \log x^{-1} .
$$

Now $\delta=\beta \gamma-\alpha=-\alpha_{1}$, and hence

$$
\begin{aligned}
\int_{0}^{1}\left(T^{*} f\right)^{p}(x) w(x) d x & \leq \beta^{p} \int_{0}^{1} x^{-\alpha_{1} p}\left(\log x^{-1}\right)^{p} f\left(x^{\beta}\right)^{p} w(x) d x \\
& \leq C \int_{0}^{1}\left(\log x^{-1}\right)^{p} f(x)^{p} w(x) d x \quad \text { by Theorem } 5.1 \\
& \leq C \int_{0}^{1} x^{-\alpha_{1} p} f(x)^{p} w(x) d x \\
& \leq C \int_{0}^{1} f^{p} w \quad \text { by Theorem } 5.1
\end{aligned}
$$

which completes the proof.

If $\alpha_{1}=\gamma_{1}=0$, we have

Theorem 5.4. If $1 \leq p<\infty, \beta=\frac{\alpha}{\gamma} \geq 1$, then (5.3) holds iff (5.2) holds and

$$
\int_{0}^{r} \log \frac{r}{x} w(x) \leq C \int_{0}^{r^{\beta}} w, \quad \forall 0<r<1 .
$$

Proof. Let $r=\psi(y)^{1 / \beta}$ in (5.5), where $\psi:(0, \infty) \rightarrow(0,1)$ is onto, and $\psi \downarrow$. Hence

$$
\int_{0}^{\infty} \int_{0}^{\psi(y)^{1 / \beta}} \log \frac{\psi(y)^{1 / \beta}}{x} w(x) d x d y \leq C \int_{0}^{1} \psi^{-1}(x) w(x) d x .
$$

By changing the order of integration, letting $t=\psi(y)$, and integrating by parts, we get (note that $\alpha_{1}=\gamma_{1}=0$ )

$$
\text { LHS }=\frac{1}{\beta} \int_{0}^{1} \int_{x^{\beta}}^{1} \psi^{-1}(t) \frac{d t}{t} w(x) d x=\frac{1}{\beta} \int_{0}^{1} T^{*} \psi^{-1}(x) w(x) d x .
$$

Take $\psi^{-1}(t)=f(t)$, we get the result for $p=1$. For $p>1$, for $f \downarrow$, let

$$
F(x)=p \beta f(x)\left[\int_{x}^{1} \frac{f(u)}{u}\right]^{p-1},
$$


then

$$
\begin{aligned}
\int_{0}^{1}\left(T^{*} f\right)^{p} w & =\int_{0}^{1} T^{*} F w \\
& \leq C \int_{0}^{1} F w \quad \text { from the case } p=1 \\
& =C \int_{0}^{1} f(x)\left[\int_{x}^{1} \frac{f(u)}{u}\right]^{p-1} w(x) d x \\
& \leq C \int_{0}^{1} f\left(T^{*} f\right)^{p-1} w \quad \text { since } \beta \geq 1
\end{aligned}
$$

We complete the proof by using Hölder's inequality.

Remark. We also mention here that for $\beta \geq 1,0<p \leq 1,(5.5)$ holds iff

$$
\int_{0}^{r}\left(\log \frac{r}{x}\right)^{p} w(x) d x \leq C \int_{0}^{r^{\beta}} w, \quad \forall 0<r<1 .
$$

In fact suppose (5.6) holds, then in Theorem 2.2 we take

$$
\phi(x, y)=\frac{1}{y} \chi_{\left(x^{\beta}, 1\right)}(y),
$$

we get

Let $f \downarrow$, and take

$$
\int_{0}^{1}\left(T^{*} f\right)^{p} w \leq C \int_{0}^{1} f^{p} w
$$

$$
F(x)=\frac{1}{p}\left(\int_{x}^{1} \frac{f(u)}{u}\right)^{\frac{1}{p}-1} f(x),
$$

then $T^{*} f(x)=\left(T^{*} F(x)\right)^{p}$, so

$$
\begin{aligned}
\int_{0}^{1} T^{*} f w & =\int_{0}^{1}\left(T^{*} F\right)^{p} w \leq C \int_{0}^{1} F^{p} w \\
& \leq \frac{C}{p^{p}} \int_{0}^{1}\left(T^{*} f\right)^{1-p} f^{p} w, \quad \text { since } \beta \geq 1 .
\end{aligned}
$$

This implies (5.5) by Hölder's inequality.

For $0<p \leq 1$, we may use Theorems 2.1 and 2.2.

\section{SPECial Weights}

For operators of the form

$$
T f(x)=\int_{0}^{\infty} \phi(t) f(t x) d t
$$

Minkowski's integral inequality easily gives us a necessary condition for a norm inequality. For special multiplicative weights the necessary condition is also sufficient. The condition will also give us examples showing that $(\mathrm{H} 1),(\mathrm{H} 4)$ are necessary for norm inequalities in Theorems 3.1 and 4.1. 
Theorem 6.1. Suppose $1 \leq p<\infty, \phi: \mathbb{R}_{+} \rightarrow \mathbb{R}_{+}, w: \mathbb{R}_{+} \rightarrow \mathbb{R}_{+}$such that $w \uparrow$ and $w(x y) \sim w(x) w(y)$. Then

$$
\|T f\|_{p, w} \leq C\|f\|_{p, w}, \quad f \downarrow
$$

iff

$$
\int_{0}^{\infty} \frac{\phi(t)}{t^{1 / p}} w(1 / t)^{1 / p} d t<\infty
$$

Proof. Note that $w(1) \sim w(x) w(1 / x)$ or $w(1 / x) \sim 1 / w(x)$.

$(6.2) \rightarrow(6.1)$.

$$
\begin{aligned}
\|T f\|_{p, w} & \leq \int_{0}^{\infty} \phi(t)\left\{\int_{0}^{\infty} f(t x)^{p} w(x) d x\right\}^{1 / p} d t \\
& =\int_{0}^{\infty} \phi(t)\left\{\int_{0}^{\infty} f(u)^{p} w(u / t) \frac{d u}{t}\right\}^{1 / p} d t \\
& \leq C \int_{0}^{\infty} \frac{\phi(t)}{[t w(t)]^{1 / p}} d t\|f\|_{p, w} .
\end{aligned}
$$

$(6.1) \rightarrow(6.2)$. For $N$ a positive integer let

$$
f_{N}(t)= \begin{cases}N^{1 / p} w(N)^{1 / p}, & \text { for } 0<t \leq 1 / N, \\ \frac{w(1 / t)^{1 / p}}{t^{1 / p}}, & \text { for } 1 / N<t \leq N, \\ 0, & \text { for } t>N\end{cases}
$$

We then have

$$
\begin{aligned}
T f_{N}(x) & =\int_{0}^{N / x} \phi(t) f_{N}(t x) d t \geq \int_{1 / N x}^{N / x} \frac{\phi(t)}{(t x)^{1 / p}} w(1 / t x)^{1 / p} d t \\
& =\frac{1}{x^{1 / p}} w(1 / x)^{1 / p} \int_{1 / N x}^{N / x} \frac{\phi(t)}{t^{1 / p}} w(1 / t)^{1 / p} d t \\
& \geq \frac{C}{[x w(x)]^{1 / p}} \int_{1 / N x}^{N / x} \frac{\phi(t)}{t^{1 / p}} w(1 / t)^{1 / p} d t \\
& \geq \frac{C}{[x w(x)]^{1 / p}} \int_{1 / N^{1 / 2}}^{N^{1 / 2}} \frac{\phi(t)}{t^{1 / p}} w(1 / t)^{1 / p} d t, \text { if } 1 / N^{1 / 2} \leq x \leq N^{1 / 2} .
\end{aligned}
$$

Hence

$$
\left(\int_{1 / N^{1 / 2}}^{N^{1 / 2}} \frac{\phi(t)}{t^{1 / p}} w(1 / t)^{1 / p} d t\right)^{p} 1 / x \leq C\left(T f_{N}\right)^{p}(x) w(x)
$$

and

$$
\int_{0}^{\infty}\left(T f_{N}\right)^{p} w \leq C \int_{0}^{\infty} f_{N}^{p} w=C\left\{\int_{0}^{1 / N} N w(N) w(t) d t+\int_{1 / N}^{N} \frac{w(t) w(1 / t)}{t} d t\right\} .
$$

The expression in $\{\cdots\} \leq C(1+2 \log N)$, since $w(N) \leq w(1 / t)$ for $0<t<$ $1 / N$ and $w(t) w(1 / t) \leq C$.

Thus for every $N$,

$$
\left(\int_{1 / N^{1 / 2}}^{N^{1 / 2}} \frac{\phi(t)}{t^{1 / p}} w(1 / t)^{1 / p} d t\right)^{p} \int_{1 / N^{1 / 2}}^{N^{1 / 2}} \frac{d x}{x} \leq C(1+2 \log N)
$$

and the second integral $=\log N$. Let $N \rightarrow \infty$. 
Remark. (i) From the proof of Theorem 6.1, the condition $w \uparrow$ can be replaced by $x w(x) \uparrow$, and $\frac{1}{r} \int_{0}^{r} w \leq C w(r), \forall r>0$ and the last condition follows from the multiplicative condition for $w$.

(ii) Let for $1<p<\infty$,

$$
\phi(t)=\frac{1}{t^{1 / p^{\prime}} \log \frac{e}{t}} \chi_{(0,1)}(t) .
$$

Then $\phi \in L^{p^{\prime}}$ and $\int_{0}^{\infty} \frac{\phi(t)}{t^{1 / p}} d t=\infty$. For this $\phi$ the operator

$$
T f(x)=\int_{0}^{\infty} \phi(t) f(t x) d t, \quad f \downarrow,
$$

is not strong- $(p, p)$ by Theorem 6.1 with $w \equiv 1$. In fact we can check that $\phi$ does not satisfy the condition $(\mathrm{H} 1)$. In this case, $\phi(x, t)=\frac{1}{x} \phi(t / x)$ and $(\mathrm{H} 1)$ becomes

$$
\Phi\left(s_{1} s_{2}\right) \leq B \Phi\left(s_{1}\right) \Phi\left(s_{2}\right), \quad \forall 0<s_{1}, s_{2}<1
$$

where $\Phi(s)=\int_{0}^{s} \phi$. Let $s_{1}=s_{2}=s$, then

$$
\frac{\Phi\left(s^{2}\right)}{\Phi(s) \Phi(s)}=\frac{\int_{0}^{s^{2}} \frac{1}{t^{1 / p^{\prime}} \log \frac{\rho}{t}} d t}{\left(\int_{0}^{s} \frac{1}{t^{1 / p^{\prime}} \log \frac{\rho}{t}} d t\right)^{2}} \rightarrow \frac{1}{2} \lim _{s \rightarrow 0} \frac{s^{1 / p}}{\int_{0}^{s} \frac{1}{t^{1 / p^{\prime}} \log \frac{\rho}{t}}} d t=\infty .
$$

Moreover we check that (3.2) holds for $w=1,1<p<\infty$. In fact

$$
\begin{aligned}
\int_{r}^{\infty} \Phi(x, r)^{p} d x & =r \int_{1}^{\infty}\left(\int_{0}^{1 / x} \frac{1}{t^{1 / p^{\prime}} \log \frac{e}{t}} d t\right)^{p} d x \\
& \leq r \int_{1}^{\infty} \frac{1}{(\log e x)^{p}}\left(\int_{0}^{1 / x} t^{-1 / p^{\prime}} d t\right)^{p} d x \\
& =\frac{p^{p}}{p-1} r .
\end{aligned}
$$

This shows that the condition $(\mathrm{H} 1)$ is needed in general for the norm inequality (3.1) to hold. Similarly if we take

$$
\phi(t)=\frac{1}{t^{1 / p^{\prime}} \log e t} \chi_{(1, \infty)}(t),
$$

then we know that the condition ( $\mathrm{H} 4)$ is needed in general for Theorem 4.1 to hold.

\section{APPLICATIONS AND SHARP CONSTANTS}

(1) Laplace transform

$$
L f(x)=\int_{0}^{\infty} e^{-x t} f(t) d t .
$$

We can take $\phi(x, t)=e^{-x t}$ in Theorems 2.1 and 2.2 to get results for $L f(x)$. Also we can use the estimate

$$
e^{-1} \int_{x}^{\infty} f\left(\frac{1}{t}\right) \frac{d t}{t^{2}} \leq L f(x) \leq\left(1+e^{-1}\right) \int_{x}^{\infty} f\left(\frac{1}{t}\right) \frac{d t}{t^{2}},
$$


and let $\phi(x, y)=\left(x / y^{2}\right) \chi_{[x, \infty)}(y)$, then we get different versions of the results for the operator $x L f(x)$ by using Theorems 2.3 and 2.4 (see also [4]).

(2) We consider the Riemann-Liouville fractional integral operator:

$$
\begin{aligned}
R_{\alpha} f(x) & =\frac{1}{x^{\alpha}} \int_{0}^{x}(x-t)^{\alpha-1} f(t) d t \\
& =\int_{0}^{1}(1-t)^{\alpha-1} f(t x) d t, \quad f \downarrow, 0<\alpha \leq 1 .
\end{aligned}
$$

We get

Proposition 7.1. Suppose $1 \leq p<\infty, 0<\alpha \leq 1$, then

$$
\int_{r}^{\infty}\left[1-\left(1-\frac{r}{x}\right)^{\alpha}\right]^{p} w \leq C_{1} \int_{0}^{r} w, \quad \forall r>0
$$

implies

$$
\int_{0}^{\infty}\left(R_{\alpha} f\right)^{p} w \leq C_{2} \int_{0}^{\infty} f^{p} w, \quad \forall f \downarrow
$$

with $C_{2} \leq\left(\left(1+C_{1}\right) / \alpha^{p}\right)^{p}$. The converse holds with $C_{1} \leq C_{2} \alpha^{p}-1$. Moreover (7.1) is equivalent with $w \in B_{p}$.

Proof. Take

$$
\phi(x, t)=\frac{1}{x^{\alpha}}(x-t)^{\alpha-1} \chi_{(0, x)}(t)
$$

in Theorem 3.1, then

$$
\Phi(x, r)= \begin{cases}\frac{1}{\alpha}, & x<r, \\ \frac{1}{\alpha}\left[1-\left(1-\frac{r}{x}\right)^{\alpha}\right], & x \geq r .\end{cases}
$$

Clearly $\Phi(x, r) \downarrow$ in $x, \forall x>r$. To check $\phi$ satisfies (H1), let $0<r<t<x$, $a=\frac{r}{x}, b=\frac{t}{x} \quad(0<a<b<1)$, then

$$
\frac{\Phi(x, t) \phi(t, r)}{\phi(x, r)}=\frac{1-(1-b)^{\alpha}}{\alpha b^{\alpha}}\left(1-\frac{1-b}{1-a}\right)^{\alpha-1} \geq \frac{1}{\alpha} A(b),
$$

where $A(b)=\frac{1-(1-b)^{\alpha}}{b}$. It is easy to see that $A\left(0^{+}\right)=\alpha, A\left(1^{-}\right)=1$ and $A(\cdot) \uparrow$ by showing that $A^{\prime}>0$. So $\inf _{0<b<1} A(b)=\alpha$. Hence

$$
\phi(x, r) \leq B \Phi(x, t) \phi(t, r), \quad 0<r<t<x,
$$

with $B=1$. This implies that (H1) holds with $B=1$. Theorem 3.1 completes the proof of the first part of the proposition. The equivalence of (7.1) and $w \in B_{p}$ is clear since $\alpha \leq A(b) \leq 1$.

We also note that this $\phi$ satisfies the other conditions in $\S 3$.

(3) If we take

$$
\phi_{\alpha}(x, y)=\frac{1}{x^{\alpha} y^{1-\alpha}} \chi_{(0, x)}(y), \quad \alpha \in(0,1],
$$

in Theorem 3.1, or

$$
\phi_{\alpha}^{*}(x, y)=\frac{1}{x^{\alpha} y^{1-\alpha}} \chi_{[x, \infty)}(y), \quad \alpha \in[0,1],
$$


in Theorem 4.1 we get the results for operators

$$
A_{\alpha} f(x)=\frac{1}{x^{\alpha}} \int_{0}^{x} f(y) \frac{d y}{y^{1-\alpha}}, \quad A_{\alpha}^{*} f(x)=\frac{1}{x^{\alpha}} \int_{x}^{\infty} f(y) \frac{d y}{y^{1-\alpha}} .
$$

(4) Define $w \in B_{\infty}^{*}$ iff

$$
\int_{0}^{r} \log \frac{r}{x} w(x) \leq C \int_{0}^{r} w
$$

Using the notation of [7], we have

Theorem 7.2. If $(u, v) \in A_{p_{0}}^{*}, 0<p<\infty, 1 \leq p_{0}<\infty, w \in B_{p / p_{0}} \cap B_{\infty}^{*}$, where $B_{p / p_{0}}=B_{p / p_{0}}\left(\phi_{\alpha}\right), \alpha=1$, then

$$
\int_{0}^{\infty}\left((M f)_{u}^{*}\right)^{p} w \leq C \int_{0}^{\infty}\left(f_{v}^{*}\right)^{p} w
$$

where

$$
(u, v) \in A_{p_{0}}^{*} \leftrightarrow \inf \left\{p:(u, v) \in A_{p}\right\}=p_{0} .
$$

This follows from Theorem 2.2, Remark after Theorem 5.4 with $\beta=1$ and methods developed in [6].

(5) We now compute some special sharp constants. We first do this for the operator $A_{\alpha} f$.

Theorem 7.3. Suppose $0<p<\infty, \alpha>0, \alpha p-s>1, s>-1$ then for $w=x^{s}$, we have

$$
C_{1} \int_{0}^{\infty} f^{p} w \leq \int_{0}^{\infty}\left(A_{\alpha} f\right)^{p} w \leq C_{2} \int_{0}^{\infty} f^{p} w, \quad \forall f \downarrow
$$

where

$$
C_{1}= \begin{cases}\alpha^{1-p} \frac{p}{\alpha p-s-1}, & p \geq 1 \\ \left(\frac{p}{\alpha p-s-1}\right)^{p}, & 0<p \leq 1\end{cases}
$$

and

$$
C_{2}= \begin{cases}\left(\frac{p}{\alpha p-s-1}\right)^{p}, & p \geq 1 \\ \alpha^{1-p} \frac{p}{\alpha p-s-1}, & 0<p \leq 1 .\end{cases}
$$

Moreover these constants are sharp.

We will prove this theorem in several lemmas.

Lemma 7.4. For $0<p<\infty, 0<\alpha<\infty, p \alpha-s>1, s>-1$, then for $w=x^{s}$,

$$
\int_{0}^{\infty}\left(A_{\alpha} f\right)^{p} w \leq C_{0} \int_{0}^{\infty} f^{p} w, \quad \forall f \downarrow
$$

where

$$
C_{0}= \begin{cases}\left(\frac{p}{\alpha p-s-1}\right)^{p}, & p \geq 1 \\ \alpha^{-p} \frac{\alpha p}{\alpha p-s-1}, & 0<p \leq 1 .\end{cases}
$$

Moreover, the constant is sharp.

Proof. Let $\phi(x, y)=x^{-\alpha} y^{\alpha-1} \chi_{(0, x)}(y)$, then

$$
\Phi(x, r)= \begin{cases}\frac{1}{\alpha}, & x<r, \\ \frac{1}{\alpha}\left(\frac{r}{x}\right)^{\alpha}, & x \geq r,\end{cases}
$$


and $B=\alpha$. So

$$
\frac{1}{\alpha^{p}} \int_{0}^{r} w+\frac{1}{\alpha^{p}} \int_{r}^{\infty}\left(\frac{r}{x}\right)^{\alpha p} w \leq C_{1} \int_{0}^{r} w, \quad \forall r>0
$$

implies (7.2) with $C_{0} \leq B^{p(p-1)} C_{1}^{p}$, by Theorem 3.1 if $p \geq 1$, and $C_{0}=C_{1}$ if $0<p \leq 1$ by Theorem 2.2. Now take $w=x^{s}$, then it is easy to compute that $C_{1}=\frac{\alpha p}{\alpha p-s-1} \alpha^{-p}$. So, we get the result for $0<p \leq 1$, and that $C_{0} \leq\left(\frac{p}{\alpha p-s-1}\right)^{p}$, if $p \geq 1$. We now show that the constant is the best for $p \geq 1$. For further use we let $0<p<\infty$, and take

$$
f_{a}(x)=x^{a-\alpha} \chi_{(0,1)}, \quad \text { for } \alpha-\frac{1+s}{p}<a<\alpha .
$$

Then

$$
A_{\alpha} f_{a}(x)= \begin{cases}\frac{1}{a} x^{a-\alpha}, & x \leq 1 \\ \frac{1}{a} \frac{1}{x^{\alpha}}, & x>1\end{cases}
$$

So

$$
\begin{aligned}
\int_{0}^{\infty}\left(A_{\alpha} f_{a}\right)^{p} w & =\int_{0}^{1} a^{-p} x^{p(a-\alpha)} x^{s} d x+a^{-p} \int_{1}^{\infty} x^{-\alpha p} x^{s} d x \\
& =\frac{a^{-p}}{1+s-(\alpha-a) p}+\frac{a^{-p}}{\alpha p-s-1}, \\
\int_{0}^{\infty} f_{a}^{p} w & =\frac{1}{1+s-(\alpha-a) p} .
\end{aligned}
$$

Thus

$$
\frac{\int_{0}^{\infty}\left(A_{\alpha} f_{a}\right)^{p} w}{\int_{0}^{\infty} f_{a}^{p} w} \rightarrow\left(\frac{p}{\alpha p-s-1}\right)^{p}
$$

by letting $a \rightarrow \frac{\alpha p-s-1}{p}$, we get $C_{0} \geq\left(\frac{p}{\alpha p-s-1}\right)^{p}$, which completes the proof.

Lemma 7.5. For $1 \leq p<\infty, \alpha>0, \alpha p-s>1, s>-1, w=x^{s}$,

$$
\int_{0}^{\infty} f^{p} w \leq \frac{\alpha p-s-1}{p} \alpha^{p-1} \int_{0}^{\infty}\left(A_{\alpha} f\right)^{p} w, \quad \forall f \downarrow .
$$

Moreover, the constant is sharp.

Proof. By Theorem 2.1 with $w=v=x^{s}, \phi(x, y)=x^{-\alpha} y^{\alpha-1} \chi_{(0, x)}(y), p=q$, we can compute the constant $C^{p}=\frac{\alpha p-s-1}{p} \alpha^{p-1}$. It is clear that the constant is sharp.

In particular, if we take $\alpha=1, s=0$, we have

Corollary 7.6. we have for $1<p<\infty$,

$$
\frac{p}{p-1} \int_{0}^{\infty} f^{p} \leq \int_{0}^{\infty}\left(\frac{1}{x} \int_{0}^{x} f(t) d t\right)^{p} d x \leq\left(\frac{p}{p-1}\right)^{p} \int_{0}^{\infty} f^{p}, \quad \forall f \downarrow .
$$

To prove next lemma, we recall the Hölder's inequality. For $0<p \leq 1$,

$$
\int f g^{p-1} \geq\left(\int f^{p}\right)^{1 / p}\left(\int g^{p}\right)^{1-1 / p} \text {. }
$$


Lemma 7.7. Suppose $0<p \leq 1, \alpha>0$, and $\forall r>0$,

$$
\int_{r}^{\infty}\left(\frac{r}{x}\right)^{\alpha p} w \geq C_{0} r w(r)
$$

then

$$
\left(p C_{0}\right)^{p} \int_{0}^{\infty} f^{p} w \leq \int_{0}^{\infty}\left(A_{\alpha} f\right)^{p} w .
$$

Note. If for some $s<\alpha p-1, x^{-s} w \uparrow$, then $w$ satisfies the hypothesis.

Proof. We have

$$
\begin{aligned}
\int_{0}^{\infty}\left(A_{\alpha} f\right)^{p} w & =p \int_{0}^{\infty} \int_{0}^{x}\left(\int_{0}^{y} \frac{f(t)}{t^{1-\alpha}}\right)^{p-1} \frac{f(y)}{y^{1-\alpha}} d y \frac{w(x)}{x^{\alpha} p} d x \\
& =p \int_{0}^{\infty} \int_{y}^{\infty} \frac{w(x)}{x^{\alpha} p} d x\left(\int_{0}^{y} \frac{f(t)}{t^{1-\alpha}}\right)^{p-1} \frac{f(y)}{y^{1-\alpha}} d y \\
& \geq p C_{0} \int_{0}^{\infty} \frac{w(y)}{y^{\alpha p-1}}\left(\int_{0}^{y} \frac{f(t)}{t^{1-\alpha}}\right)^{p-1} \frac{f(y)}{y^{1-\alpha}} d y \\
& =p C_{0} \int_{0}^{\infty} f\left(A_{\alpha} f\right)^{p-1} w .
\end{aligned}
$$

The proof is completed by applying Hölder's inequality.

Lemma 7.8. Suppose $0<p \leq 1, \alpha p-s>1, s>-1$, and $w(x)=x^{s}$, then

$$
\left(\frac{p}{\alpha p-s-1}\right)^{p} \int_{0}^{\infty} f^{p} w \leq \int_{0}^{\infty}\left(A_{\alpha} f\right)^{p} w
$$

Proof. Take $w(x)=x^{s}$ in Lemma 7.7, then $C_{0}=\frac{1}{\alpha p-s-1}$. The proof of Lemma 7.4 also shows that this constant is sharp.

Note that the above two lemmas hold for all $f$ not only for $f \downarrow$. Now combining Lemmas 7.4,7.5, and 7.8 we complete the proof for Theorem 7.3.

Next we compute some best constants for the operator $A_{\alpha}^{*} f$.

Theorem 7.9. Suppose $0<p<\infty, \alpha \geq 0, \alpha p-s<1$, then for $w=x^{s}$, we have

$$
C_{1} \int_{0}^{\infty} f^{p} w \leq \int_{0}^{\infty}\left(A_{\alpha}^{*} f\right)^{p} w \leq C_{2} \int_{0}^{\infty} f^{p} w, \quad \forall f \downarrow
$$

where

$$
\begin{aligned}
& C_{1}= \begin{cases}\frac{p}{\alpha^{p}} B\left(p, \frac{1+s}{\alpha}-p\right), & p \geq 1, \alpha>0, \\
\frac{p}{(1+s)^{p}} \Gamma(p), & p \geq 1, \alpha=0, \\
\left(\frac{p}{1+s-\alpha p}\right)^{p}, & 0<p \leq 1,\end{cases} \\
& C_{2}= \begin{cases}\left(\frac{p}{1+s-\alpha p}\right)^{p}, & p \geq 1, \\
\frac{p}{\alpha^{p}} B\left(p, \frac{1+s}{\alpha}-p\right), & 0<p \leq 1, \alpha>0, \\
\frac{p}{(1+s)^{p}} \Gamma(p), & 0<p \leq 1, \alpha=0,\end{cases}
\end{aligned}
$$

and $B(\cdot, \cdot)$ is the beta function. Moreover these constants are sharp.

We will also prove this theorem in several lemmas. 
Lemma 7.10. For $0<p \leq 1, \alpha p-s<1, w=x^{s}$,

(i) if $\alpha>0$,

$$
\int_{0}^{\infty}\left(A_{\alpha}^{*} f\right)^{p} w \leq c_{0} \int_{0}^{\infty} f^{p} w, \quad \forall f \downarrow
$$

where $c_{0}=\alpha^{-p} p B\left(p, \frac{1+s}{\alpha}-p\right)$.

(ii) if $\alpha=0$,

$$
\int_{0}^{\infty}\left(\int_{x}^{\infty} \frac{f(t)}{t} d t\right)^{p} w d x \leq \frac{p}{(1+s)^{p}} \Gamma(p) \int_{0}^{\infty} f^{p} w, \quad \forall f \downarrow .
$$

(iii) In particular for $\alpha=1, s=0$, since $B(p, 1-p)=\Gamma(p) \Gamma(1-p)=\frac{\pi}{\sin \pi p}$, we have for $0<p<1$,

$$
\int_{0}^{\infty}\left(\frac{1}{x} \int_{x}^{\infty} f(t) d t\right)^{p} d x \leq \frac{\pi p}{\sin \pi p} \int_{0}^{\infty} f(x)^{p} d x, \quad \forall f \downarrow
$$

Moreover all constants are sharp.

Proof. In Theorem 2.2, let $\phi(x, y)=x^{-\alpha} y^{\alpha-1} \chi_{(x, \infty)}(y)$, then for $r \geq x$,

$$
\Phi(x, r)= \begin{cases}\frac{1}{\alpha}\left(\left(\frac{r}{x}\right)^{\alpha}-1\right), & \alpha>0 \\ \log \frac{r}{x}, & \alpha=0\end{cases}
$$

Hence for $\alpha>0$,

$$
\begin{aligned}
C^{p} & =\frac{1}{\alpha^{p}} \frac{s+1}{r^{s+1}} \int_{0}^{r}\left(\left(\frac{r}{x}\right)^{\alpha}-1\right)^{p} x^{s} d x \\
& =\frac{s+1}{\alpha^{1+p}} \int_{0}^{1}(1-y)^{p} y^{\frac{1+s}{\alpha}-1-p} d y \\
& =\frac{s+1}{\alpha^{1+p}} B\left(\frac{1+s}{\alpha}-p, p+1\right) \\
& =\alpha^{-p} p B\left(p, \frac{1+s}{\alpha}-p\right) .
\end{aligned}
$$

For $\alpha=0$,

$$
C^{p}=\frac{1+s}{r^{1+s}} \int_{0}^{r}\left(\log \frac{r}{x}\right)^{p} x^{s} d x=(1+s) \int_{0}^{\infty} t^{p} e^{-(s+1) t} d t=(1+s)^{-p} p \Gamma(p) .
$$

Hence we complete the proof.

Lemma 7.11. Suppose $\alpha \geq 0, p \geq 1$ and $\forall r>0$,

$$
\int_{0}^{r}\left(\frac{r}{x}\right)^{\alpha p} w(x) d x \leq C_{0} r w(r)
$$

then

$$
\int_{0}^{\infty}\left(A_{\alpha}^{*} f\right)^{p} w \leq\left(p C_{0}\right)^{p} \int_{0}^{\infty} f^{p} .
$$

Note. If for some $s>\alpha p-1, x^{-s} w \uparrow$, then $w$ satisfies the hypothesis. 
Proof. We have

$$
\begin{aligned}
\int_{0}^{\infty}\left(A_{\alpha}^{*} f\right)^{p} w & =p \int_{0}^{\infty} \int_{x}^{\infty}\left(\int_{y}^{\infty} \frac{f(t)}{t^{1-\alpha}} d t\right)^{p-1} \frac{f(y)}{y^{1-\alpha}} d y \frac{w(x)}{x^{\alpha p}} d x \\
& =p \int_{0}^{\infty}\left(\int_{y}^{\infty} \frac{f(t)}{t^{1-\alpha}} d t\right)^{p-1} \frac{f(y)}{y^{1-\alpha}} \int_{0}^{y} \frac{w(x)}{x^{\alpha p}} d x d y \\
& \leq C_{0} p \int_{0}^{\infty}\left(\int_{y}^{\infty} \frac{f(t)}{t^{1-\alpha}} d t\right)^{p-1} f(y) y^{\alpha(1-p)} w(y) d y \\
& =C_{0} p \int_{0}^{\infty}\left(A_{\alpha}^{*} f\right)^{p-1} f w .
\end{aligned}
$$

We complete the proof by applying Hölder's inequality.

Lemma 7.12. Suppose $\alpha \geq 0, p \geq 1, \alpha p-s<1, w(x)=x^{s}$ then

$$
\int_{0}^{\infty}\left(A_{\alpha}^{*} f\right)^{p} w \leq\left(\frac{p}{1+s-\alpha p}\right)^{p} \int_{0}^{\infty} f^{p} w .
$$

Moreover, the constant is sharp.

Proof. In Lemma 7.11, let $w(x)=x^{s}$, then we can take $C_{0}=\frac{1}{1+s-\alpha p}$, so (7.3) holds. We now show the constant is the best. For $0<p<\infty$, let

$$
f_{a}=x^{-(a+\alpha)} \chi_{(0,1)}, \quad 0<a<\frac{1+s-\alpha p}{p} .
$$

Then

$$
A_{\alpha}^{*} f_{a}(x)=\frac{1}{x^{\alpha}} \int_{x}^{1} t^{-a-1} d t=\frac{1}{a x^{\alpha}}\left(\frac{1}{x^{a}}-1\right)
$$

So

$$
\begin{aligned}
\int_{0}^{\infty}\left(A_{\alpha}^{*} f_{a}\right)^{p} w & =a^{-p} \int_{0}^{1} x^{-\alpha p+s}\left(\frac{1}{x^{a}}-1\right)^{p} d x \\
\int_{0}^{\infty} f_{a}^{p} w & =\int_{0}^{1} x^{-(a+\alpha) p+s} d x \\
& =\frac{1}{1+s-(a+\alpha) p} .
\end{aligned}
$$

Let $\beta=1+s-(a+\alpha) p>0$, we have

$$
\begin{aligned}
\frac{\int_{0}^{\infty}\left(A_{\alpha}^{*} f_{a}\right)^{p} w}{\int_{0}^{\infty} f_{a}^{p} w} & =(1+s-(a+\alpha) p) a^{-p} \int_{0}^{1}\left(1-x^{a}\right)^{p} x^{s-(\alpha+a) p} d x \\
& =a^{-p} \beta \int_{0}^{1}\left(1-x^{a}\right)^{p} x^{\beta-1} d x \\
& =a^{-p} p a \int_{0}^{1} x^{\beta}\left(1-x^{a}\right)^{p-1} x^{a-1} d x \\
& \rightarrow a_{0}^{1-p} p \int_{0}^{1}\left(1-x^{a_{0}}\right)^{p-1} x^{a_{0}-1} d x=a_{0}^{-p},
\end{aligned}
$$

by the dominated convergence theorem, and letting $a \rightarrow a_{0} \equiv \frac{1+s-\alpha p}{p}$, or $\beta \rightarrow$ 0 . 
Lemma 7.13. Suppose $0<p \leq 1, \alpha \geq 0$, and $\forall r>0$,

$$
\int_{0}^{r}\left(\frac{r}{x}\right)^{\alpha p} w \geq C_{0} r w(r)
$$

then

$$
\left(p C_{0}\right)^{p} \int_{0}^{\infty} f^{p} w \leq \int_{0}^{\infty}\left(A_{\alpha}^{*} f\right)^{p} w .
$$

Note. If for some $s>\alpha p-1, x^{-s} w \downarrow$, then $w$ satisfies the hypothesis.

Proof. We have

$$
\begin{aligned}
\int_{0}^{\infty}\left(A_{\alpha}^{*} f\right)^{p} w & =p \int_{0}^{\infty} \int_{x}^{\infty}\left(\int_{y}^{\infty} \frac{f(t)}{t^{1-\alpha}}\right)^{p-1} \frac{f(y)}{y^{1-\alpha}} d y \frac{w(x)}{x^{\alpha p}} d x \\
& =p \int_{0}^{\infty} \int_{0}^{y} \frac{w(x)}{x^{\alpha p}} d x\left(\int_{y}^{\infty} \frac{f(t)}{t^{1-\alpha}}\right)^{p-1} \frac{f(y)}{y^{1-\alpha}} d y \\
& \geq p C_{0} \int_{0}^{\infty} \frac{w(y)}{y^{\alpha p-1}}\left(\int_{y}^{\infty} \frac{f(t)}{t^{1-\alpha}}\right)^{p-1} \frac{f(y)}{y^{1-\alpha}} d y \\
& =p C_{0} \int_{0}^{\infty} f\left(A_{\alpha}^{*} f\right)^{p-1} w .
\end{aligned}
$$

The proof is completed by applying Hölder's inequality.

Lemma 7.14. Suppose $0<p \leq 1, \alpha p-s<1, \alpha \geq 0$, then for $w=x^{s}$, we have

$$
\left(\frac{p}{1+s-\alpha p}\right)^{p} \int_{0}^{\infty} f^{p} w \leq \int_{0}^{\infty}\left(A_{\alpha}^{*} f\right)^{p} w
$$

Moreover the constant is sharp.

Proof. Take $w=x^{s}$, in Lemma 7.13, then $C_{0}=\frac{1}{1+s-\alpha p}$. The proof of Lemma 7.12 shows that this constant is sharp.

Note that the above four lemmas hold for any $f$ not only for $f \downarrow$.

Lemma 7.15. Suppose $p \geq 1, \alpha p-s<1, \alpha \geq 0$, then for $w=x^{s}$,

$$
C_{0} \int_{0}^{\infty} f^{p} w \leq \int_{0}^{\infty}\left(A_{\alpha}^{*} f\right)^{p} w, \quad \forall f \downarrow
$$

where

$$
C_{0}= \begin{cases}\frac{p}{\alpha^{p}} B\left(p, \frac{1+s}{\alpha}-p\right), & p \geq 1, \alpha>0, \\ \frac{p}{(1+s)^{p}} \Gamma(p), & p \geq 1, \alpha=0 .\end{cases}
$$

Moreover the constant is sharp.

Proof. Take $w=v=x^{s}, p=q$ in the Theorem 2.1, then the same computation as in the proof of Lemma 7.10 gives the constant $C_{0}$. Clearly the constant is the best.

Finally combining Lemmas $7.10,7.12,7.14$, and 7.15 we get Theorem 7.9. 


\section{REFERENCES}

1. M. Ariño and B. Muckenhoupt, Maximal functions on classical Lorentz spaces and Hardy's inequality with weights for non-increasing functions, Trans. Amer. Math. Soc. 320 (1990), 727-735.

2. C. Bennett and R. Sharpley, Interpolation of operators, Math. 129, Academic Press, 1988.

3. M. Sh. Braverman, On a class of operators, preprint.

4. H. P. Heinig, Weighted inequalities in Fourier analysis, preprint.

5. M. A. Leckband and C. J. Neugebauer, A general maximal operator and the $A_{p}$-condition, Trans. Amer. Math. Soc. 275 (1983), 821-831.

6. C. J. Neugebauer, Weighted norm inequalities for averaging operators of monotone functions, Publ. Mat. (to appear).

7. C. J. Neugebauer, Some classical operators on Lorentz spaces, Forum Mat. (to appear).

8. E. Sawyer, Boundedness of classical operators on classical Lorentz spaces, Studia Math. 96 (1990), 145-158.

9. D. Stepanov, The weighted Hardy's inequality for nonincreasing operators of monotone functions, preprint.

Department of Mathematics, Purdue University, West Lafayette, Indiana 47907

E-mail address: lai@math.purdue.edu 